\title{
Micro- and Nanostructured Devices for the Investigation of Biomolecular Interactions
}

\author{
Christophe Danelon ${ }^{\mathrm{a}}$, Martin G. Jenke ${ }^{\mathrm{b}}$, Christoph Schreitera, Gyu Man Kim ${ }^{\mathrm{b}, \mathrm{c}}$, Jean-Baptiste \\ Perez $^{\mathrm{a}}$, Christian Santschi ${ }^{\mathrm{b}, \mathrm{d}}$, Jürgen Brugger ${ }^{\mathrm{b}}$, and Horst Vogel ${ }^{\star a}$
}

\begin{abstract}
Cell membrane receptors and ion channels are essential in many different cellular processes. To analyze the activity of membrane proteins in vesicles and biological cells, we fabricated micro-nanostructured chips, enabling the application of electrophysiology and fluorescence-based techniques. A SU-8 biochip was developed to simultaneously micromanipulate and investigate optically and electrically individual vesicles in a microfluidic channel. Lipid vesicles were transported, positioned by electrophoretic movement on a micrometer sized aperture, and fused to form a planar suspended membrane, which is suited to study ion channel activity. Aiming to investigate $G$ protein coupled receptor signaling pathways in native-like environment, we developed a method for producing well-oriented planar cell membrane sheets on silicon films containing nanoaperture arrays. The accessibility of extracellular and cytosolic surfaces was demonstrated by targeting membrane constituents side-specifically with fluorescent markers. Our approach can be applied for studying membrane proteins from a large variety of cells and cellular organelles using chip-based screening assays.
\end{abstract}

Keywords: Electrophysiology · Fluorescence techniques · Membrane proteins · Micro-nanoapertures

\section{Introduction}

The plasma membrane of any cell acts as a barrier that separates intracellular from extracellular space. It protects the cell from environmental influences such as osmotic pressure and damaging agents. Every cell must also be able to exchange many different substances with its environment, and to sense and react to external chemical and physical signals, which implies the transmission of information from the outside

${ }^{*}$ Correspondence: Prof. Dr. H. Vogel ${ }^{\mathrm{a}}$

Tel.: +41216933155

Fax: +41216936190

E-Mail: horst.vogel@epfl.ch

aLaboratory of Physical Chemistry of Polymers and Membranes

Ecole Polytechnique Fédérale de Lausanne (EPFL) $\mathrm{CH}-1015$ Lausanne

bMicrosystems Laboratory

Ecole Polytechnique Fédérale de Lausanne

$\mathrm{CH}-1015$ Lausanne

'Present address: School of Mechanical Engineering

Kyungpook National University

Kyungpook, South Korea

Centre Suisse d'Electronique et de

Microtechnique (CSEM)

CH-2007 Neuchâtel to the inside of the cell, across the plasma membrane.

Biomembranes contain special integrated proteins that allow the translocation of all the necessary substances. These proteins operate either by actively binding and transporting the ion or molecule, a process requiring energy, or by forming a simple diffusion pore. Examples are ion channels, which play essential roles in cellular processes such as changing the transmembrane potential, propagating the action potential, or water channels, which are responsible for osmoregulation, and are therefore influenced by many therapeutic compounds [1][2].

One of the largest classes of cell surface receptors is the $G$ protein coupled receptors (GPCRs), which transduce external stimuli across the cell membrane and thereby induce intracellular responses. There are many different types of physical and chemical signals that activate GPCRs: light, peptides, hormones, odorants, tastants, nucleotides are a few examples [3] The signal transduction process involves at least three events: the detection of the signal by the receptor (absorbing a photon or binding a ligand), a subsequent conformational change of the receptor which activates the receptor and thereby transmits the signal across the membrane, and then the binding of a suitable heterotrimeric $\mathrm{G}$ protein (composed of three subunits named $\alpha, \beta$ and $\gamma$ ) to the activated receptor, which in turn ac- tivates the G protein. GPCRs play a major role in the perception and the detection of light and smell. It was recently estimated that about $50 \%$ of the clinically used drugs target GPCRs [4].

Here, we present novel techniques for functional analysis of ion channels and membrane receptors. The first part describes the principles of electrophysiological measurements with planar lipid membranes for single ion channel analysis. The second part presents the development of a microfluidic chip, made of SU-8 photoresist, suited to manipulate vesicles and biological cells to investigate the activity of ion channels in a 'planar patch clamp' configuration. The third part concerns a novel procedure for transferring cell membrane fragments onto micro- and nanostructured surfaces offering substantial advantages for fluorescencebased investigation of complex signaling processes at the plasma membrane such as those mediated by GPCRs.

\section{Functional Analysis of Ion Channels}

\subsection{Single Channel Analysis with Standard Electrophysiology Techniques}

Electrophysiological techniques are ideally suited to study the function of membrane channels with single molecule reso- 
lution. Under an applied transmembrane voltage, ion currents flowing through channel proteins either reconstituted in an artificial planar lipid bilayer or present in the plasma membrane of a biological cell are recorded [5]. The patch-clamp technique was developed to investigate the activity of ion channels in their native cell membrane environment [6]. In a typical experiment, a stable, electrically insulating seal is formed between the tip of a glass micropipette and the cell membrane. Ion currents passing the protein channels in the patch of the plasma membrane under the micropipette can be recorded with single channel resolution.

In a black lipid membrane (BLM) experiment [7-9], a planar lipid bilayer is formed across an aperture in a thin film of electrically insulating material separating two aqueous compartments (Fig. 1A). Into each compartment an electrode is inserted to apply a transmembrane voltage, up to $100-200 \mathrm{mV}$, and to record the ionic current flowing through channel proteins reconstituted into the BLM. Easy access to both sides facilitates direct electrical measurement and manipulation of the composition of the bathing solutions. There are a number of well-established protocols of how to reconstitute membrane proteins into BLMs [9]:

i) Formation of BLMs using lipid monoor bilayers, which already contain the particular channel protein;

ii) Fusion of lipid vesicles comprising reconstituted channel proteins to a preformed BLM;

iii) Purified, detergent solubilized channel proteins are added to the chamber and spontaneously insert into the preformed BLM.

Under applied voltage, insertion of the channel proteins into BLMs (ii \& iii) is easily detected as a current jump resulting from the flow of ions through the channel. Finally, the behavior of the channel protein is studied under various conditions, for instance by changing the transmembrane potential (voltage-gated ion channels), adding a ligand (ligand-gated ion channels), a permeating molecule or an ion.

The ion current fluctuation induced by the transient occupancy of the pore by a diffusing molecule is an electrical signature of the molecular interactions occurring inside the protein channel [10]. The magnitude of the current decrease reflects the extent of channel block by the translocating molecule, while the duration of the current pulse refers to the strength of interactions between the molecule and the amino acids lining the pore. This approach, known as the molecular Coulter counter, provides rapid and accurate characterization in realtime of targeted analytes interacting with single ion channels [11][12]. The passage of carbohydrates [13], nucleic acids [14],
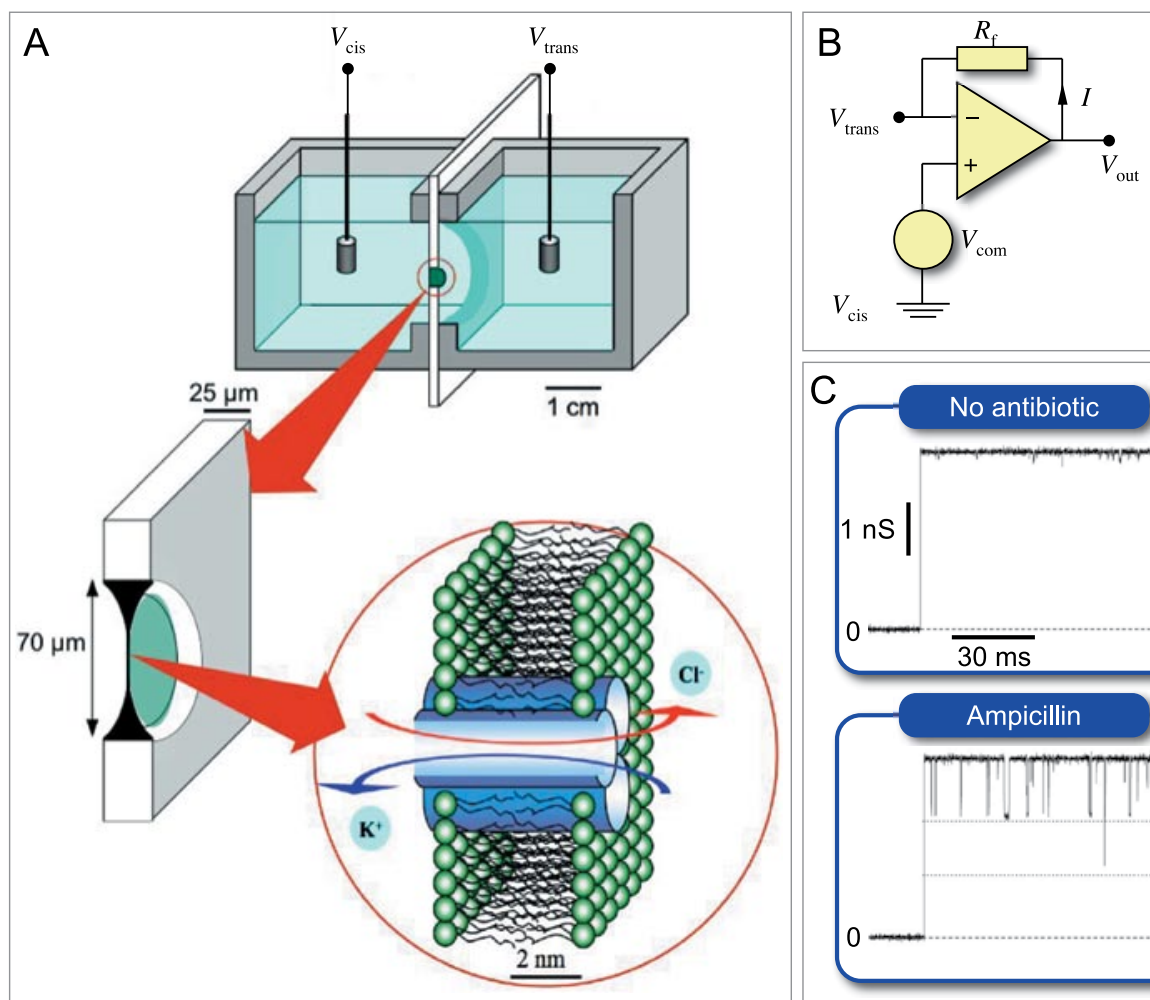

Fig. 1. Principle of BLM experiments. A) A $25 \mu \mathrm{m}$ thick Teflon film containing a $60 \mu \mathrm{m}$ diameter aperture separates two compartments containing an electrolyte solution. A planar lipid bilayer is formed across the aperture. The electrical properties of the system are measured with two electrodes, typically Ag/ $\mathrm{AgCl}$ electrodes. B) The electronic circuit allows simultaneously to apply a constant potential $V_{\text {com }}$ to the trans electrode ( $V_{\text {trans }} \equiv V_{\text {com }}$, and $V_{\text {cis }}$ is grounded), as well as to measure the current $I$ flowing through the feedback resistance $R_{\mathrm{f}}\left(/ / V\right.$ conversion with $R_{\mathrm{f}}$ in the order of $\left.1 \mathrm{G} \Omega\right)$. The output potential $V_{\text {out }}$ is equal to I. $R_{\mathrm{f}}$ after subtracting the $V_{\text {com }}$ component in a second amplifier. C) Protein channels injected in the aqueous solution spontaneously insert in the planar membrane generating a constant ionic current that results from the flow of ions across the water-filled pores spanning the bilayer. When a single OmpF porin is reconstituted, the diffusion of ampicillin molecules inside the pores is seen as transient current interruptions. Because the amplitude, the duration and frequency of blocking events are specific to each antibiotic, the microscopic mechanism of translocation can be related to the structure and physicochemical properties of the drugs.

ATP molecules [15], antibiotics [16][17], could be resolved on a single molecular level.

In the example shown in Fig. 1C, a single bacterial porin OmpF (Outer Membrane Protein F) of Escherichia coli has been reconstituted into a planar lipid bilayer. The OmpF channel is considered to be the main pathway for $\beta$-lactam antibiotics to cross the bacterial outer membrane into the periplasmic space [18]. The crystallographic structure reveals a homotrimeric organization with three water-filled channels spanning the membrane [19]. A single porin spontaneously inserts into the lipid membrane introducing a constant ionic current. Addition of ampicillin, a drug of choice for the treatment of $E$. coli infections, gives rise to time-resolved current interruptions [16]. Ampicillin blocks the OmpF conductance by $1 / 3$ corresponding to the complete closure of one monomer in the channel trimer. The average residence time of an ampicillin molecule in the pore is about $150 \mu \mathrm{s}$, which suggests strong interactions between the penetrating drug and
OmpF channel [16]. Therefore, the capacity of different antibiotics to pass through single OmpF channels can be probed and their efficiency to penetrate bacteria can be rationalized in terms of basic molecular interactions [17].

\subsection{Planar Patch Clamp within SU-8 Microchannels}

Although traditional patch clamp and planar lipid bilayer techniques are valuable tools for fundamental research in ion channels, high-throughput screening of pharmaceutical compounds requires automation and miniaturization on the microand nanometer scale [20][21]. In the planar patch clamp approach, a planar microchip containing an aperture is used to replace the classical pipette [22][23]. A variety of materials can be employed for the fabrication of patch clamping devices such as glass [24], silicon [22] [23], and polydimethylsiloxane (PDMS) [25]. This method enables automated and high quality functional analysis of even single ion channels from mammalian cells as well as lipid vesicles. 


\subsubsection{Materials, Design and Experimental Set-up}

Recently, we described the fabrication and application of a bioanalytical chip, made of SU-8 photoresist, comprising integrated, high aspect ratio microfluidic channels, suitable to manipulate and investigate vesicles and biological cells [26]. A central micrometer-sized aperture allows planar membrane experiments. Microfluidics allow (sub)micrometer-sized objects to be transported and addressed with different chemicals by means of pressure control and electrophoretic movement. The new chip was designed for combined optical and electrophysiological measurements.

The chip was fabricated using SU-8 as material [27], which enables a highly flexible manufacturing of micrometer scale devices. SU-8 is a transparent, negative-tone, epoxy-type photoresist that can be spincoated and structured in layers that are a few hundred nanometers up to $2 \mathrm{~mm}$ thick. Negative images of an exposure mask can be made in the SU-8 layer by means of standard UV-lithography $(365 \mathrm{~nm}$ wavelength exposure) and allow outstanding aspect ratios up to $25: 1$. SU-8 is biocompatible and chemically resistant to most organic solvents and many acids [28]. Its surface is negatively charged in aqueous solution, becomes hydrophobic in air but can be reactivated by oxygen plasma and kept hydrophilic in water

We exploited these characteristics to fabricate a biochip entirely made of SU-8 that consists of a central thin film, a microfluidic channel network and a central micrometer-sized aperture, as shown schematically in Fig. 2. The central aperture can be used to sort vesicles or to position them for subsequent biophysical and bioanalytical investigations, such as fluorescence imaging or electrophysiological measurements. The channel configuration enables very convenient liquid handling and submicrometer precise positioning of vesicles (and cells). The channel height is given by one of the SU-8 layers and can be varied from 50-100 $\mu \mathrm{m}$ compatible for microscopic investigations with objectives of high numerical aperture (up to NA = 1.2).

The main steps for the fabrication of microstructured SU-8 chips are:

i) Vacuum deposition of a 200-nm-thick chrome and a 500-nm-thick aluminum sacrificial layer by evaporation on polished silicon wafer;

ii) Spin coating, prebake, exposure and post exposure bake of three consecutive SU-8 layers, $3.3 \mu \mathrm{m} / 20 \mu \mathrm{m} / 50 \mu \mathrm{m}$ thick, respectively;

iii) Development of the exposed triple SU-8 layer;

iv) Surface releasing by electrochemical etching of aluminum.
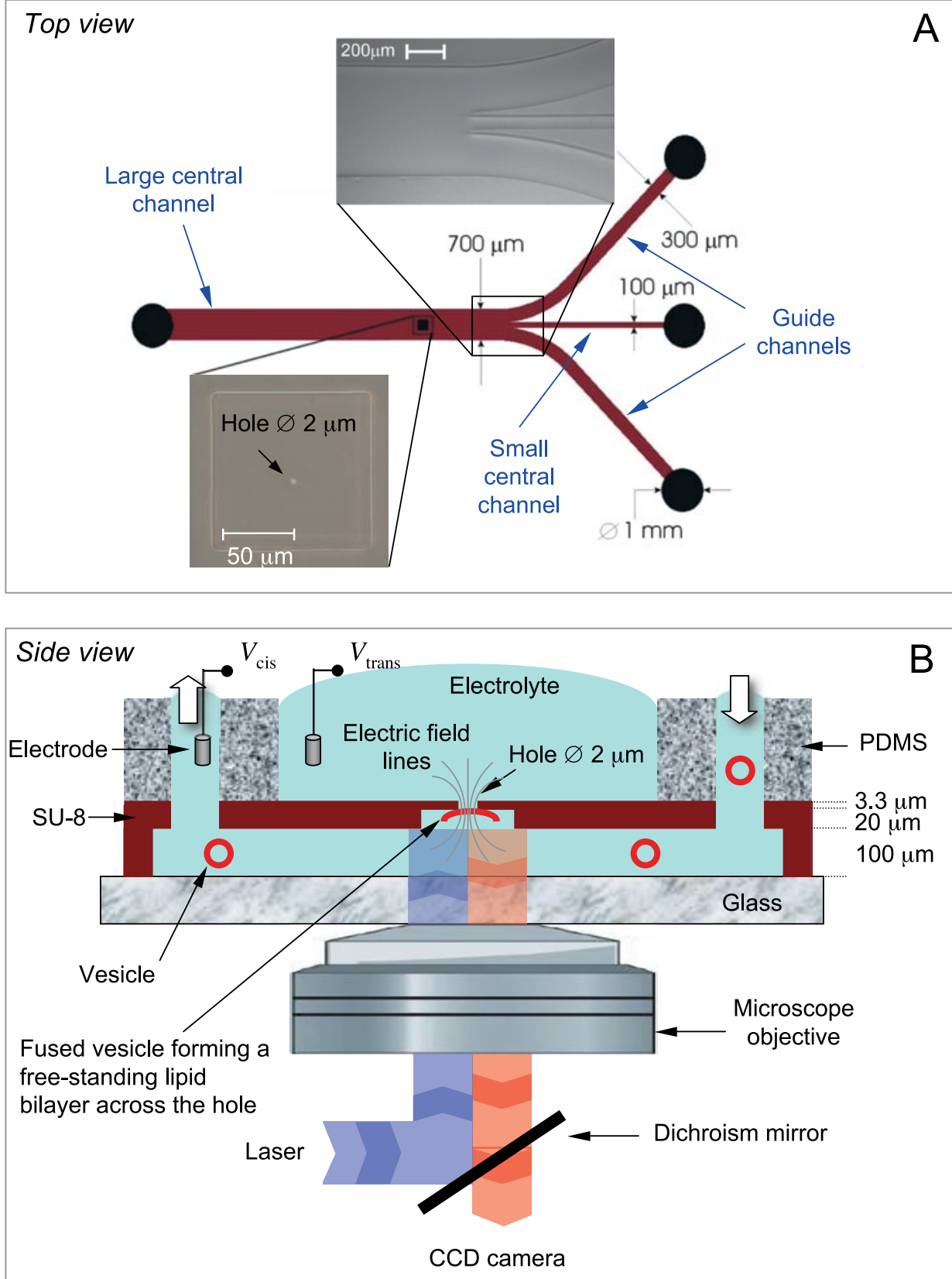

Fig. 2. Schematic view of the SU-8 chip. A) Top-view of the chip showing the three inlet channels (guide channels and small central channel) convening to form one large central channel. A micrometersize aperture is fabricated in the large central channel. B) Cross sectional view of the large central channel at the aperture together with the glass slide that seals the microfludic network and the optical microscope objective below. The PDMS block serves as sealing material and provides access holes for the buffer solutions to the microchannels and to the top-side of the chip. Lipid vesicles containing a fluorescent membrane marker are injected in the microchannel and directed to the aperture with pressure and electrophoretic controls.

The microfabricated and released biochips were mounted 'sandwich-like' on a $170 \mu \mathrm{m}$ thick standard microscope slide, sealed by a PDMS pad, and fixed with a polymethyl methacrylate (PMMA) plate that was screwed to the microscope holder. The PDMS pad served as sealing material and spacer, and provided access holes for the fluids and the electrodes. Holes in the PMMA plate allowed the fluidic connection to a pump comprising 10-25 $\mu \mathrm{l}$ syringes for automatic fluidic delivery and pressure control.
Giant unilamellar vesicles (GUVs) were prepared by overnight swelling dried lipid films of $80 \%$ 1,2-dioleoyl-sn-glycero3-phosphatidylcholine (DOPC), 20\% 1-palmitoyl-2-oleoyl-sn-glycero-3-phosphatidylglycerol (POPG) (Avanti Polar Lipids, USA) in $10 \mathrm{ml}$ of $200 \mathrm{mM}$ sorbitol solution. For fluorescence detection, lipid vesicles were labeled with $0.5 \%$ of $\mathrm{N}-(6-$ tetramethylrhodaminethiocarbamoyl)-1,2dihexadecanoyl-sn-glycero-3-phosphatidylethanolamine (TRITC-DHPE) (Molecular Probes, USA). Giant unilamellar 
vesicles had typical sizes up to $60 \mu \mathrm{m}$ diameter and were stored at $4{ }^{\circ} \mathrm{C}$.

For vesicle or cell positioning, the chip was placed at an inverted fluorescence microscope (Zeiss, Germany) equipped with a $\mathrm{Hg} / \mathrm{Xe}$ lamp and adequate filters for exciting and observing rhodamine fluorescence (excitation $545 \mathrm{~nm}$, emission $570 \mathrm{~nm}$ ). Images were taken with a FAC 830 CCD camera (Grundig, Germany) in phase contrast using $10 \times$ and $40 \times$ Zeiss objectives. For planar membrane characterization, we used an inverted scanning confocal microscope (Zeiss LSM510) equipped with a 63× water immersion objective of $1.2 \mathrm{NA}$ and $240 \mu \mathrm{m}$ working distance (Zeiss).

\subsubsection{Vesicle Positioning and Planar Membrane Formation}

The fabricated chip consists of three inlet channels and one outlet channel. The design of the chip (Fig. 2) and the laminar flow characteristics allow the middle inlet channel flow to transport the vesicles or chemical compounds under study directly over the fabricated central hole. The flow behavior in the chip was observed by light transmission and fluorescence microscopy, injecting water into the central channel and an aqueous solution of rhodamine into the neighboring guide channels. By varying the inlet flow rate and pressure of each of the three channels, it was possible to modulate the width of the central flow between 20 and $80 \mu \mathrm{m}$, and to laterally displace the central flow by up to $300 \mu \mathrm{m}$ from the central axis of the chip [26]. The flow speed could be varied from 0.1 to $50 \mathrm{~mm} / \mathrm{s}$ without changing the flow characteristics of the system. In this way, the microfluidic system could be used as a perfusion system to rapidly apply chemical compounds such as ligands to a target vesicle trapped on the hole.
The same flow principle was applied to guide GUVs at low speed $(0.1 \mathrm{~mm} / \mathrm{s})$ towards the membrane and the hole. Fine positioning onto the hole was done by electrophoretic movement. Three chlorinated silver rods integrated in the PDMS block were used as electrodes. The laminar flow was stopped when a suitable vesicle arrived near the membrane and a voltage between 10 and $15 \mathrm{~V}$ was then applied to position with submicrometer precision a vesicle to the central hole (Fig. 3A).

Upon contact with the chip surface, the vesicle opens and spreads forming a planar lipid bilayer spanning the aperture (Fig. 3B). Micrometer-sized apertures improve the mechanical stability of the lipid bilayer by designing a hybrid configuration between solid supported and free-standing membranes. The chip surface pretreated with positively charged polymers provides stable planar membranes with giga-seals, which is suited for single channel recording [22]. The planar geometry of the chip and the microfluidic channel allow the combination with fluorescence-based single molecule detection. As a future development, recording chips with multiple apertures can be used for parallel measurements.

\section{Planar Cell Membranes for Imaging Complex Biomolecular Interactions}

Signaling pathways mediated by membrane receptors such as GPCRs rely on a complex biomolecular network involving several integral and membrane-anchored proteins. In vitro studies of such processes implies that all membrane constituents taking part in the signaling cascade are maintained in their native supramolecular organization to ensure their functional- ity. Isolation and immobilization of native membrane fragments derived from living cells provide an alternative approach to artificial lipid membranes since no purification and reconstitution steps of the different compounds are required.

\subsection{Preparation and Visualiza- tion Strategies of Supported Cell Membrane Sheets}

Different procedures have been developed to deposit native cell membranes on planar surfaces and beads. Plasma membrane fragments prepared by sonication or homogenization were immobilized by sedimentation to investigate the organization and structure of membrane proteins by atomic force microscopy [29] and electron microscopy [30], but the orientation of the membrane relative to the support is not controlled. Inside-out membrane sheets (the inner leaflet of the plasma membrane is exposed to the solution) were obtained by adhesion of living cells followed by sonication [31], lysis [32] or pressure-induced cell rupture [33]. Spontaneous fusion of erythrocytes on micropatterned surfaces has also been reported [34].

Recently, we showed that large membrane sheets $\left(>100 \mu \mathrm{m}^{2}\right)$ can be detached from the plasma membrane of adherent living cells and transferred to a poly-L-lysine (PLL)-coated glass slide [33][35] or a micro- nanostructured silicon chip [36]. The composition of the original cell membrane is preserved as well as the fluidity of both membrane leaflets [33]. The proteins of interest expressed at the plasma membrane were visualized as fusion constructs with fluorescent proteins [33][35][36].

Cell membrane sheets were prepared from human embryonic kidney (HEK) cells expressing the $\alpha 1 b$-adrenergic receptor $(\alpha 1 b-A R)$ [33] or the neurokinin-1 recep-

\section{A}
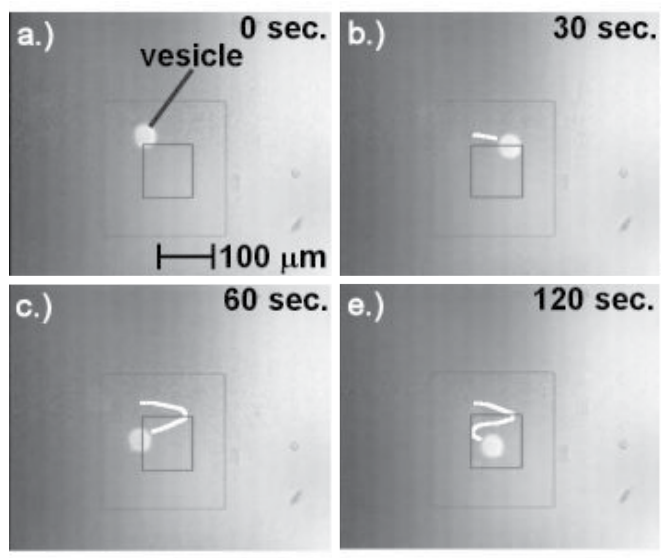

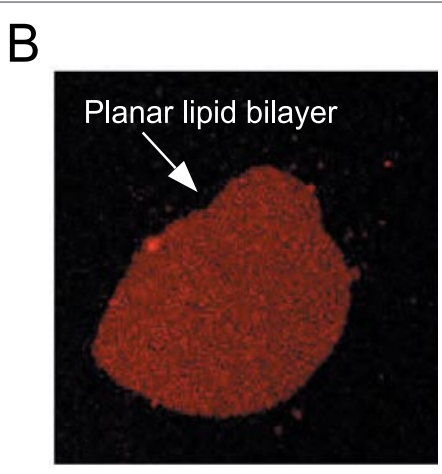

Fluorescence

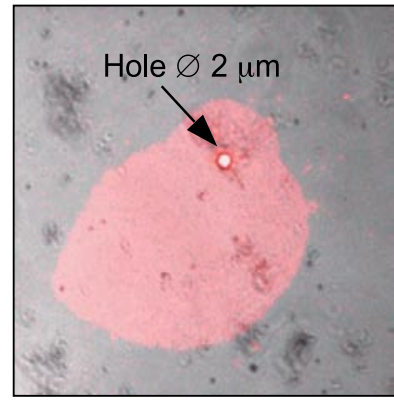

Fluorescence

$+$

Transmitted light

Fig. 3. A) Directing a negatively charged lipid vesicle towards the central hole by applying an electric potential of $10 \mathrm{~V}$ between top and bottom electrodes. Images (a)-(d): Trajectory (white line drawn artificially) of the vesicle during its passage towards the central hole within a time period of two minutes. The $3.3 \mu \mathrm{m}$ thick SU-8 film is highlighted by a rectangle. B) Scanning fluorescence images of a lipid vesicle positioned on the aperture and spread as a planar membrane on the PLL-coated SU-8 surface. 

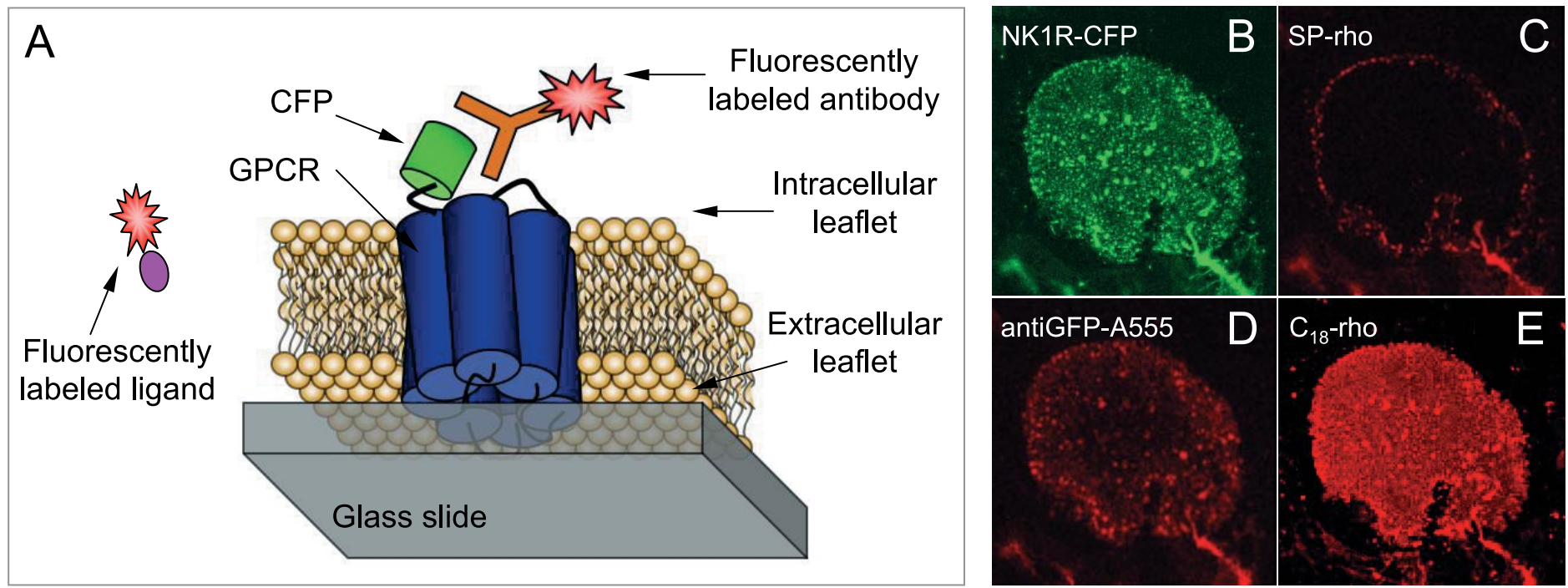

Fig. 4. Characterization of native membrane patches and analysis of receptor accessibility by scanning confocal microscopy. A) After transfer of cell membrane fragments on a glass slide, the cytosolic side of GPCRs is fully accessible while the extracellular side faces the support. B) Cell membrane sheets containing the NK1R-CFP are immobilized on a glass coverslip. C) The membrane patches were incubated with the agonist substance $P$ fluorescently labeled with rhodamine (SP-rho) $50 \mathrm{nM}$ for $15 \mathrm{~min}$. D) $20 \mathrm{~min}$ incubation with the antibody anti-GFP labeled with Alexa Fluor-555 (antiGFP-A555) 5 nM. E) Subsequent addition of the membrane dye $\mathrm{C}_{18}$-rho $100 \mathrm{nM}$

tor (NK1R) [36] as prototypical GPCRs. The $\alpha 1 b-A R$ and the NK1R were genetically fused at their intracellular C-terminus with the green fluorescent protein (GFP) and the cyan fluorescent protein (CFP), respectively, yielding the fusion receptors $\alpha 1 \mathrm{~b}-\mathrm{AR}-\mathrm{GFP}$ and NK1R-CFP. Membrane sheets containing the recombinant fluorescent receptors were imaged by scanning confocal microscopy.

\subsection{Accessibility and Functionality of Integral Membrane Proteins}

Fig. 4B shows a large membrane fragment of HEK cells expressing the NK1RCFP immobilized on a PLL-coated glass slide. Addition of the membrane dye $\mathrm{C}_{18}$-rho leads to a nearly homogeneous labeling of the cell fragment indicating that the non-fluorescent areas in Fig. 4B do not correspond to membrane defects but rather to low-density receptor regions (Fig. 4E). Membrane sheets with regions of higher fluorescence intensity as shown in Fig. 4E were occasionally observed and might correspond to trafficking vesicles stuck at the plasma membrane.

As illustrated in Fig. 4A and Fig. $5 \mathrm{~A}, \mathrm{C}$, the orientation of membrane proteins relative to the surface is controlled by our procedure of transferring membrane sheets: the extracellular membrane leaflet is facing the support, while the cytoplasmic leaflet is exposed to the bulk aqueous phase. The accessibility of the cytosolic side of the receptors was investigated by using the C-terminal fluorescent protein as recognition element (Fig. 4A). Addition of the fluorescent antibody antiGFP-Alexa Fluor-555 (antiGFP-A555) leads to complete labeling of the planar membranes demonstrating that the cytosolic part of the receptor is fully accessible (Fig. 4D). Therefore, supported membrane sheets are suited to investigate internal membrane processes [35].

Investigating the functionality of receptors in the supported cell membrane sheets requires unrestricted access of the extracellular binding site for ligand molecules. With the $\alpha 1 b-A R$, the related antagonist prazosin (and its fluorescent derivative prazosin-Bodipy FL) and agonist epinephrine are membrane permeable, which allowed the conduction of ligand binding experiments [33]. However, the ability of a compound to cross the cell membrane depends on its physico-chemical properties and in most cases the permeability is limited.

As shown in Fig. 4C with cell membrane sheets containing the NK1R, addition of the fluorescent derivative of the agonist substance P (SP-rho) leads to a crown-like labeling of the patches. This result indicates that only the binding sites of the peripheral receptors are accessible. The signal can be displaced in an excess of unlabeled SP demonstrating the binding specificity of SP-rho to the NK1R. Even after $2 \mathrm{~h}$ incubation with SP-rho, we could not observe the labeling of the whole patch suggesting that the fluorescent ligand cannot diffuse across the cell membrane in the time range of the experiments.

\subsection{Planar Cell Membranes Suspended Across Nanoaperture Arrays}

In order to extend pharmacological studies to membrane impermeable li- gands, cell membrane sheets were transferred on a planar support structured with arrays of submicrometer-sized apertures (Fig. 5A-C), which provides full accessibility to the intra- and extracellular sides of the membranes suspended across the holes [36].

We used silicon technology as a suitable approach for nanopore fabrication [37] and to integrate artificial and cell membranes [22][23]. A square silicon chip with a 100-500 nm thick central freestanding SiN film was engineered with arrays of holes with 50-600 $\mathrm{nm}$ diameter by electron beam lithography and focused ion beam. Fig. 5B shows a scanning electron micrograph of $500 \mathrm{~nm}$ diameter holes arrayed in a $5 \mu \mathrm{m}$ quadratic lattice. The procedure for preparing planar suspended cell membranes is the same as previously described. The main steps are illustrated in Fig. 5A. After transferring the cell membrane sheets on the aperture arrays, the silicon chip is mounted into a PDMS chamber comprising a microchannel for fluidic exchange. The horizontal configuration of the device and the narrow bottom channel are designed for fluorescence microscopy with short working distance objectives.

Cell membrane sheets larger than 100 $\mu \mathrm{m}^{2}$ can regularly be deposited onto the aperture arrays. Formation of suspended planar cell membranes depends on the dimension and arrangement of the apertures. Yields are high for pores with diameters $<500 \mathrm{~nm}$ and separated by $5 \mu \mathrm{m}$. Typical immobilized cell membranes containing the NK1R-CFP are shown in Fig. 6. The periodicity of the chip structure facilitates the localization of the nanopores covered 


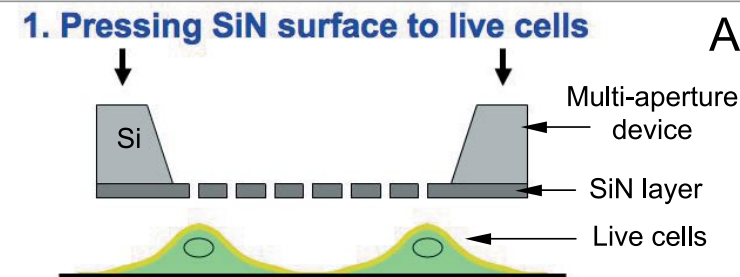

2. Transfer of cell membrane fragments
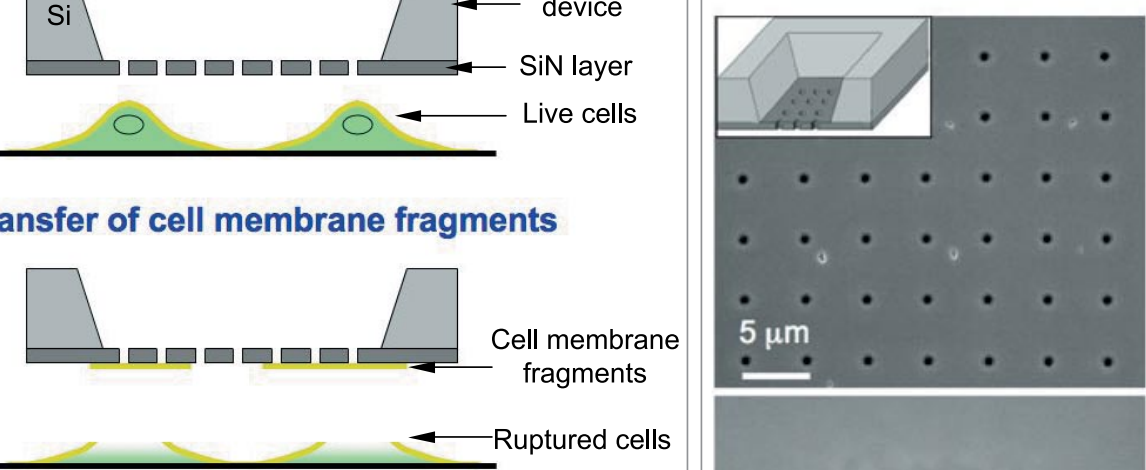

\section{Integration of fluidic channel}
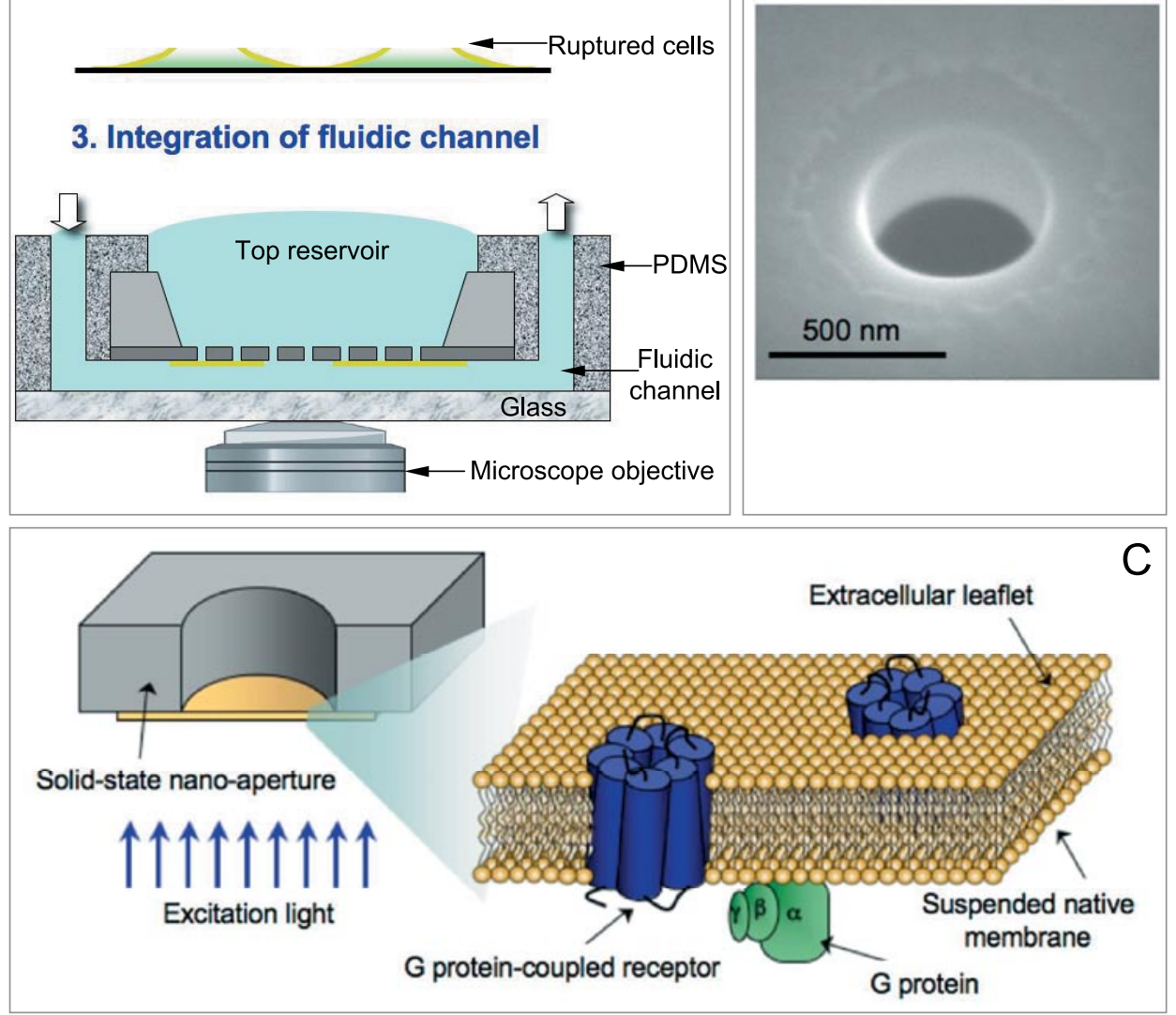

Fig. 5. A) Procedure for the preparation of suspended cell membrane sheets. Native membrane fragments are transferred onto the nanopore arrays by first pressing the chip surface to a supported layer of cultured cells and then rupturing the cells by removing the chip with the attached upper cell membrane fragments. Finally, the chip is integrated into a PDMS microfluidic chamber suitable for optical imaging. B) Scanning electron micrographs of $5 \mu \mathrm{m}$ lattice array (top) of $500 \mathrm{~nm}$ holes fabricated in a $500 \mathrm{~nm}$ thick SiN film (bottom). (Inset, top) Scheme of the 3D structure of the silicon chip. (C) Schematic view of a planar native membrane covering a nanoaperture. Reprinted with permission from [36]. Copyright (2006) American Chemical Society.

by the membrane sheets. No alteration of the membrane morphology due to the presence of the apertures is detected within the spatial resolution of the confocal microscope. Intact supported planar cell membranes could be observed over days after transfer demonstrating the long-term stability of the cell membranes suspended across the holes.

To demonstrate that the extracellular surface of the cell membrane sheets could be accessed by specific compounds diffusing across the nanopores, we added from the top reservoir a derivative of the stricted access for aqueous solutions and analytes from the pore side. In the future we will use more sensitive fluorescence techniques such as fluorescence correlation spectroscopy and single molecule imaging to investigate ligand binding to the extracellular side of the few receptors present in the nanopores.

\section{Perspectives}

Our results on the transport and micromanipulation of vesicles in microchannels should find broad bioanalytical applications for artificial or native cell-derived vesicles. Because the laminar flows can be rapidly displaced across an immobilized vesicle, it is possible to apply various chemical compounds, comparable to classical stoppedflow experiments, but here at ultrasmall volumes down to attoliters. After vesicle spreading as planar membranes across the aperture, the activity of ion channels can be investigated with simultaneous optical and electrical detection [38-40]. Of particular interest would be to investigate ligandgated ion channels such as the 5HT3 and the nicotinic acetylcholine receptors.

We believe that the cell membrane sheets, suspended across nanopores, have a potential for a number of applications in the field of miniaturized bioanalytical and biophysical investigations of membrane processes:

i) Optical (fluorescence) microscopy to detect molecular reactions with single molecule resolution at, in and across cell membranes. Here, the low fluorescence background due to the absence of the cytosol allows single molecule imaging of lipids and proteins with an exceptional signal-to-noise ratio [33]. Of particular interest in this context are novel, selective protein labeling techniques, which allow the introduction of stable fluorophores in living cells [41-43].

ii) Planar patch-clamp measurements to detect electrically single channel activities in combination with fluorescence techniques [38-40].

iii) Scanning probe techniques such as atomic force microscopy to monitor the transduction of external signals across the cell membrane in terms of protein structural changes and lateral organization.

iv) Attachment of living cells across nanopore arrays for local dispensing of activating compounds with submicrometer sized lateral distribution.

v) Biosensing on a large variety of cells and cellular organelles due to the longterm stability of planar native membranes. 


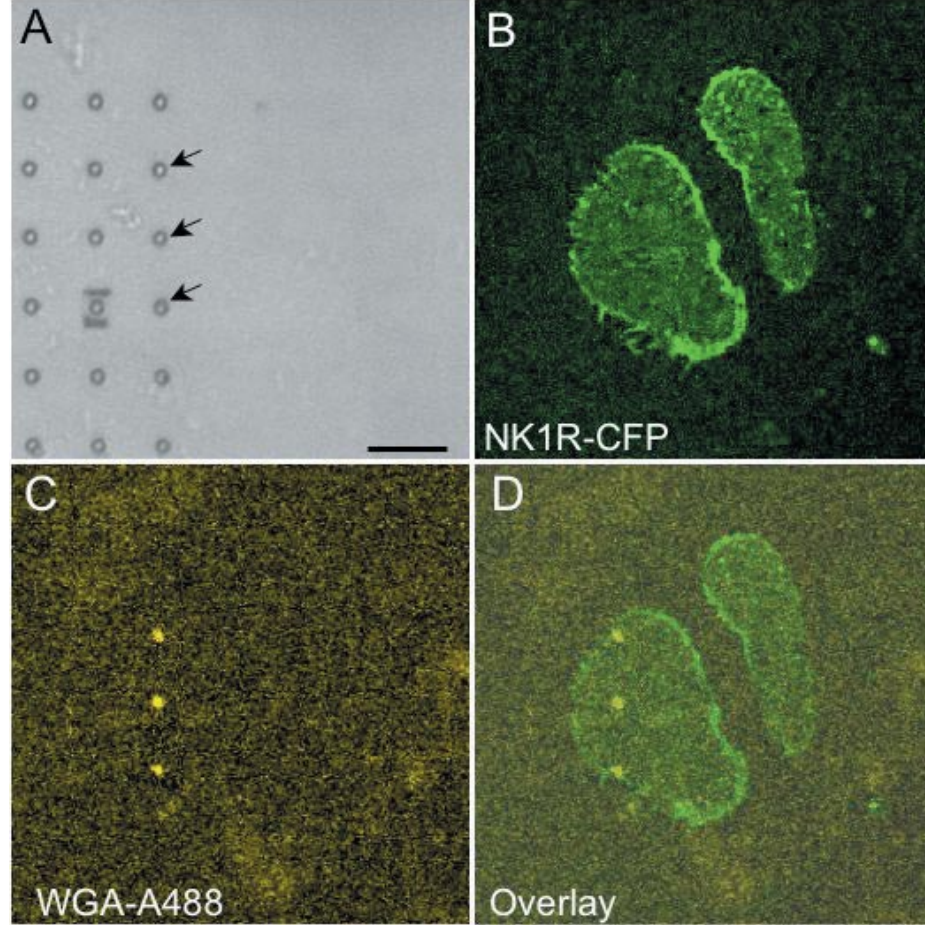

Fig. 6. Scanning confocal images of two cell membrane sheets expressing the fluorescent NK1R-CFP. Cell membranes were immobilized on the SiN support across $500 \mathrm{~nm}$ apertures arrayed on a $5 \mu \mathrm{m}$ quadratic lattice. A) The SiN support is transparent as observed by the transmitted light. The arrows indicate the holes covered by a membrane sheet. The scale bar is $5 \mu \mathrm{m}$. B) Cell membrane sheets are imaged by the CFP fluorescence of the receptors. C) Fluorescence microscopy after few minutes incubation of a solution of $5 \mu \mathrm{g} / \mathrm{ml}$ WGA-A488 in the top reservoir followed by extensive washing with buffer. D) Overlay of the NK1R-CFP and WGA-A488 fluorescence signals. The lower compartment was continuously washed with buffer during the incubation of WGA-A488 on top. Reprinted with permission from [36]. Copyright (2006) American Chemical Society.

\section{Acknowledgements}

This work was supported by the European Commission via contract LSHG-CT-2004504601 (E-MeP), the TopNano21 programme and the EPFL. We thank Sébastien Jiguet for his assistance with the $\mathrm{SU}-8$ process.

Received: August 29, 2006

[1] B. Hille, 'Ion channels of excitable membranes' 3nd edn. Sinauer, Sunderland, 2001.

[2] F.M. Ashcroft, 'Ion channels and disease' Academic Press, San Diego, 1999.

[3] T.H. Ji, M. Grossmann, I. Ji, J. Biol. Chem. 1998, 273, 17299.

[4] A.D. Howard, G. McAllister, S.D. Feighner, Q. Liu, R.P. Nargund, L.H. Van der Ploeg, A.A. Patchett, Trends Pharmacol. Sci. 2001, 22, 132.

[5] B. Sakmann, E. Neher, 'Single-channel recording' 2nd edn. Plenum Press, New York, 1995.

[6] E. Neher, B. Sakmann, Nature 1976, 260, 799.

[7] M. Montal, P. Mueller, Proc. Natl. Acad. Sci. USA 1972, 69, 3561.

[8] H. Schindler, FEBS Lett. 1980, 122, 77.

[9] W. Hanke, W.-R. Schlue, 'Planar lipid bi- layers: methods and applications' Academic Press, San Diego, 1993.

[10] S.M. Bezrukov, I. Vodyanoy, V.A. Parsegian, Nature 1994, 370, 279.

[11] S.M. Bezrukov, J. Membrane Biol. 2000 174,1

[12] H. Bayley, C.R. Martin, Chem. Rev. 2000, $100,2575$.

[13] S.M. Bezrukov, L. Kullman, M. Winterhalter, FEBS Lett. 2000, 476, 224.

[14] J.J. Kasianowicz, E. Brandin, D. Branton, D.W. Deamer, Proc. Natl. Acad. Sci. USA 1996, 93, 13770.

[15] T.K. Rostovtseva, S.M. Bezrukov, Biophys. J. 1998, 74, 2365

[16] E.M. Nestorovich, C. Danelon, M. Winterhalter, S.M. Bezrukov, Proc. Natl. Acad. Sci. USA 2002, 99, 9789.

[17] C. Danelon, E.M. Nestorovich, M. Winterhalter, M. Ceccarelli, S.M. Bezrukov, Biophys. J. 2006, 90, 1617.

[18] H. Nikaido, Microbiol. Mol. Biol. Rev. 2003, 67, 593.

[19] S.W. Cowan, T. Schirmer, G. Rummel, M. Steiert, R. Ghosh, R.A. Pauptit, J.N. Jansonius, J.P. Rosenbusch, Nature 1992, $358,727$.

[20] X. Wang, M. Li, Assay Drug Dev. Technol. 2003, 5, 695.
[21] C. Wood, C. Williams, G.J. Waldron, Drug Discov. Today 2004, 9, 434

[22] C. Schmidt, M. Mayer, H. Vogel, Angew. Chem., Int. Ed. 2000, 39, 3137.

[23] N. Fertig, A. Tilke, R.H. Blick, J.C. Behrends, G. ten Bruggencate, J.P. Kotthaus, Appl. Phys. Lett. 2000, 77, 1218.

[24] N. Fertig, C. Meyer, R.H. Blick, C. Trautmann, J.C. Behrends, Phys. Rev. E 2001 , 64, 040901.

[25] K.G. Klemic, J.F. Klemic, M.A. Reed, F.J Sigworth, Biosens. Bioelectron. 2002, 17, 597.

[26] M.G. Jenke, C. Schreiter, G.M. Kim, H Vogel, J. Brugger, Microfluid. Nanofluid. in press.

[27] K.Y. Lee, N. LaBianca, S.A. Rishton, S. Zolgharnain, J.D. Gelorme, J. Shaw, T.H.P. Chang, J. Vac. Sci. Technol. B 1995, 13, 3012 .

[28] M.O. Heuschkel, L. Guerin, B. Buisson, D. Bertrand, P. Renaud, Sens. Actuators $B$ 1998, 48, 356.

[29] Y. Liang, D. Fotiadis, S. Filipek, D.A. Saperstein, K. Palczewski, A. Engel, J. Biol. Chem. 2003, 278, 21655.

[30] I. Prior, C. Muncke, R. Parton, J. Hancock, J. Cell. Biol. 2003, 160, 165.

[31] B.S. Jacobson, D. Branton, Science 1977, 195, 302

[32] N. Hirai, S. Sonobe, T. Hayashi, Proc. Natl. Acad. Sci. USA 1998, 95, 15102.

[33] J.-B. Perez, K.L. Martinez, J.-M. Segura, H. Vogel, Adv. Funct. Mater. 2006, 16 , 306.

[34] M. Tanaka, A.P. Wong, F. Rehfeldt, M. Tutus, S. Kaufmann, J. Am. Chem. Soc. 2004 126, 3257

[35] J.-B. Perez, J.-M. Segura, D. Abankwa, K.L. Martinez, H. Vogel, J. Mol. Biol., in press.

[36] C. Danelon, J.-B. Perez, C. Santschi, J. Brugger, H. Vogel, Langmuir 2006, 22 , 22.

[37] J. Li, D. Stein, C. McMullan, D. Branton, M.J. Aziz, J.A. Golovchenko, Nature 2001, 412, 166.

[38] T. Ide, Y. Takeuchi, T. Yanagida, Single Molecules 2002, 3, 33.

[39] V. Borisenko, T. Lougheed, J. Hesse, E. Fureder-Kitzmuller, N. Fertig, J.C. Behrends, G.A. Woolley, G.J. Schutz, Biophys. J. 2003, 84, 612

[40] G.S. Harms, G. Orr, M. Montal, B.D Thrall, S.D. Colson, H.P. Lu, Biophys. J. 2003, 85, 1826.

[41] E. Guignet, R. Hovius, H. Vogel, Nat. Biotechnol. 2004, 22, 440 .

[42] A. Keppler, H. Pick, C. Arrivoli, H. Vogel, K. Johnsson, Proc. Natl. Acad. Sci. USA 2004, 101, 9955

[43] B.H. Meyer, J.-M. Segura, K.L. Martinez, R. Hovius, N. George, K. Johnsson, H. Vogel, Proc. Natl. Acad. Sci. USA 2006, 103 2138. 